KUL-TF-98/31

hep-th/9808074

\title{
Anomalous D-brane and orientifold couplings from the boundary state
}

\author{
Ben Craps ${ }^{1,2}$, Frederik Roose ${ }^{3}$
}

Instituut voor theoretische fysica

Katholieke Universiteit Leuven, B-3001 Leuven, Belgium

\begin{abstract}
We compute scattering amplitudes involving both R-R and NS-NS fields in the presence of a D-brane or orientifold plane. These provide direct evidence for the anomalous couplings in the D-brane and orientifold actions. The D9-brane and O9-plane are found to couple to the first Pontrjagin class with the expected relative strength.
\end{abstract}

\footnotetext{
1 Aspirant FWO, Belgium

${ }^{2}$ E-mail: Ben.Craps@fys.kuleuven.ac.be

${ }^{3}$ E-mail: Frederik.Roose@fys.kuleuven.ac.be
}

PACS 11.25.-w, 04.65.+e 


\section{Introduction}

Since their introduction in string theory D-branes have attracted an ever increasing interest. Accordingly the effective action describing the low energy dynamics acquired various additional terms. Among these the Wess-Zumino couplings to the RR-potentials appear to be the least well-studied. Apart from the natural coupling to the $(p+1)$-form there are also interaction terms coupling a $\mathrm{D} p$-brane to e.g. the $(p-1)$ - and $(p-3)$-forms, for $p$ high enough. In [1] the precise form of these was derived from anomaly arguments:

$$
S_{W Z}=\frac{T_{p}}{\kappa} \int_{p+1} \hat{\mathcal{C}}^{\prime} \wedge e^{2 \pi \alpha^{\prime} F+\hat{B}^{\prime}} \wedge \sqrt{\hat{A}(\hat{R})}
$$

The $p_{1}(\hat{R})$ term in the expansion was already proposed in [2] based on an independent duality reasoning. In Ref. [3] a similar coupling was proposed for orientifold planes. Although void of world brane dynamics, these planes do couple to bulk fields for anomalies to cancel along the lines of Ref. [1]. In this paper we will give additional evidence for some of these terms by evaluating the corresponding string diagrams. []

Scattering amplitudes in the presence of D-branes have hitherto been evaluated in two ways; people have either essentially performed a disk or annulus calculation [4, 5] or relied on the boundary state formalism [6, 7]. We opt for the last possibility although in principle the calculations could be done analogously to [5]. A nice feature of our choice is that the results thus obtained carry over to the orientifold case almost without effort.

In section 2 we set up the basics of doing string tree level scattering involving the boundary state. As a working example we derive the $\int B \wedge \mathcal{C}_{p-1}$ coupling. In section 3 we outline the procedure to find the desired $\int p_{1} \wedge \mathcal{C}_{p-3}$ term, thereby relying on the basic results in the preceding section. Making contact with the effective D-brane action is done in section 4 . In section 5 we derive the tree level amplitude for the gravitational coupling of an orientifold plane. Section 6 then concludes and raises a few questions concerning the origin of the studied interaction terms. An appendix explaining our conventions and normalizations is added.

\section{The $B \wedge \mathcal{C}_{p-1}$ interaction}

Various string amplitude calculations [5, 7] established the coupling of a D p-brane to the $\mathrm{RR}(p-1)$-form potential:

$$
2 \sqrt{2} \pi \alpha^{\prime} T_{p} \int \mathcal{C}_{p-1} \wedge F
$$

* For explanation of the used symbols and conventions see Appendix A.

†The $p_{1}(\hat{R})$ terms in Refs [2, 3] actually came with the proposal to do the corresponding three point string amplitudes. 
where $\kappa^{2}=8 \pi G_{N}$. From the fact that only the $2 \pi \alpha^{\prime} F+B^{\prime}$ combination is gauge invariant one can then infer the presence of

$$
\sqrt{2} T_{p} \int \mathcal{C}_{p-1} \wedge \hat{B}^{\prime}
$$

This D-brane coupling to the NS-NS two form should reproduce the leading behaviour at low energies of the following string amplitude $f$ :

$$
\mathcal{A}=\left\langle\mathcal{C}_{p-1} ; k_{2}\left|V_{B}^{(0)}(\zeta, k)\right| B\right\rangle_{\mathrm{R}}
$$

which is equivalent with the two point function on the disk of the Kalb-Ramond field $B$ and the RR-potential $\mathcal{C}_{p-1}$. Here and in the following we adopt both the RRstate $\left\langle\mathcal{C}_{p-1}\right|$ and the boundary state $\left.|B\rangle_{\mathrm{R}}\right]_{\text {from }}[6]$ (and we give the RR potential a momentum $k_{2}$.). In the following we carry out the evaluation of this simplelooking amplitude using the boundary state. Thus we will find out about the basic manipulations involved when computing any boundary state amplitude.

First, as the boundary state and the RR potential already saturate the superghost anomaly [6] any additional vertex must be in the (0,0)-picture. Therefore we may take (see Eq. (A.58))

$$
V_{B}^{(0)}=\frac{\kappa}{\sqrt{2} \pi} \zeta_{\mu \nu} \int d^{2} z\left(\partial X^{\mu}+\mathrm{i} k \cdot \psi \psi^{\mu}\right)\left(\bar{\partial} X^{\nu}+\mathrm{i} k \cdot \tilde{\psi} \tilde{\psi}^{\nu}\right)
$$

where $z$ and $\bar{z}$ run over the complement of the unit disk in the complex plane.

A substantial amount of work in computing amplitudes involving the boundary state goes into pulling the non-zero mode operators

$$
\begin{aligned}
& \exp \left[-\sum_{n=1}^{\infty} \frac{1}{n} \alpha_{-n} \cdot S \cdot \tilde{\alpha}_{-n}\right] \\
& \exp \left[\mathrm{i} \eta \sum_{m=1}^{\infty} \psi_{-m} \cdot S \cdot \tilde{\psi}_{-m}\right]
\end{aligned}
$$

from the boundary state to the left where they annihilate the out vacuum. For amplitudes in the RR sector one then has to take care of the fermion zero modes. From Eq. (A.35) in the Appendix it is clear that those zero modes are effectively replaced by $\gamma$-matrices. In the conventions we are using any amplitude in the $R R$ sector contains a factor

$$
\left(\frac{1}{\sqrt{2}}\right)^{n} \operatorname{tr}\left(C^{-1} \mathcal{M} R C^{-1} \mathcal{N}^{T} L\right),
$$

where $L$ is a product of $\gamma$-matrices corresponding to the left-over left-moving fermions, $R$ a product of $\gamma$ 's for the right-moving fermions and $n$ is the total number

\footnotetext{
${ }^{\ddagger}$ with polarizations chosen along the brane worldvolume.

${ }^{\S}$ For the precise form of the RR state and the boundary state see also Appendix A.
} 
of $\gamma$ 's in $L R$. The matrices $\mathcal{M}$ and $\mathcal{N}$ show up in the boundary state and the RR state respectively. Note that Eq. (2.8) is schematic: in fact, keeping track of all factors of $\gamma_{11}$ and minus-signs is crucial to obtain correct results.

Equivalently [4], one modifies the Green functions to include left-right contractions:

$$
\begin{aligned}
\left\langle X^{\mu}(z) \bar{X}^{\nu}(\bar{w})\right\rangle & =-S^{\mu \nu} \log \left(1-\frac{1}{z \bar{w}}\right) \\
\left\langle\psi^{\mu}(z) \bar{\psi}^{\nu}(\bar{w})\right\rangle_{R} & =\frac{S^{\mu \nu}}{2} \frac{\mathrm{i} \eta}{z \bar{w}-1} \frac{1+z \bar{w}}{\sqrt{z \bar{w}}}
\end{aligned}
$$

Before applying the above rules to the amplitude Eq. (2.4), let us remark that the ghost sector is discussed in the Appendix, whereas the superghost sector contributes a factor $1 / 2$ [6].

Contracting two fermions using the massive oscillators in the boundary statef", leads to

$$
\begin{aligned}
\mathcal{A}= & \frac{T_{p}}{2} \frac{\kappa}{\sqrt{2} \pi} \frac{1}{2 \sqrt{2}(p-1) !} \frac{32}{4} \frac{1}{2} \epsilon^{\alpha_{1} \ldots \alpha_{p-1} \beta_{1} \beta_{2}} c_{\alpha_{1} \ldots \alpha_{p-1}} \zeta_{\beta_{1} \beta_{2}} \\
& \int_{|z|>1} d^{2} z\left(|z|^{2}\right)^{-k \cdot S \cdot k-k \cdot k_{2}}\left(|z|^{2}-1\right)^{k \cdot S \cdot k} \frac{(k \cdot S \cdot k)}{|z|^{2}\left(|z|^{2}-1\right)}
\end{aligned}
$$

where $c_{\alpha_{1} \ldots \alpha_{p-1}}$ is the polarization tensor of the RR field. Doing the $\int d^{2} z$ integral gives $\pi(k \cdot S \cdot k) B\left(1+k \cdot k_{2}, k \cdot S \cdot k\right)$. We thus conclude that, for small momenta,

$$
\mathcal{A}=\frac{\kappa T_{p}}{2(p-1) !} \epsilon^{\alpha_{1} \ldots \alpha_{p-1} \beta_{1} \beta_{2}} c_{\alpha_{1} \ldots \alpha_{p-1}} \zeta_{\beta_{1} \beta_{2}}
$$

We end this section with a remark which is related to the previous footnote. Because of the decoupling of longitudinal polarizations, the amplitude vanishes if $k$ (or equivalently $k^{\prime}$ ) has a non-zero component along the brane directions In computing cross-sections, one has to average over neighbouring momenta. So in order to have a non-vanishing cross-section, one needs the possible momentum components along the brane to be discrete, i.e. all worldvolume directions should be compactified. This suggests considering Euclidean branes. In that case one can have on-shell momenta without a component along the brane worldvolume. For such momenta the numerator of the expression in the footnote vanishes.

\footnotetext{
9 The zero-mode contribution is proportional to $\frac{k \cdot S \cdot k}{k \cdot k_{2}}$ for small on-shell momenta. Further investigations seem to suggest that this vanishes whenever the $B \wedge \mathcal{C}_{p-1}$ interaction could contribute to cross-sections, as we argue in the next paragraph. We thank Rodolfo Russo for pointing out this problem to us.

"This was pointed out by Igor Pesando.
} 


\section{The $\operatorname{tr} R^{2} \wedge \mathcal{C}_{p-3}$ interaction}

We have to calculate the following amplitude:

$$
\mathcal{A}=\left\langle\mathcal{C}_{p-3} ; k_{2}\left|V_{g}^{(0)}\left(\zeta_{3}, k_{3}\right) V_{g}^{(0)}\left(\zeta_{4}, k_{4}\right)\right| B\right\rangle_{\mathrm{R}}
$$

Since we are looking for the $\operatorname{tr} R^{2} \wedge \mathcal{C}_{p-3}$ term in the D-brane effective action, we will consider the components of $\mathcal{C}_{p-3}$ with indices along the brane. This implies that the fermion zero modes have to provide the four gamma-matrices with Lorentz indices in the worldvolume directions complementary to the ones of the RR potential that is being considered.

As the computation is analogous to the one presented in the previous section but considerably longer, we only give a rough sketch. Since each graviton vertex operator can be written as the sum of four different parts, the amplitude splits into various pieces. Some integrations by parts in the relative angle are needed to let the various terms combine nicely. Performing the trace of gamma-matrices, taking into account the superghost sector and the GSO-projection, one ends up with the following result (possibly up to a global sign):

$$
\begin{aligned}
\mathcal{A}= & \frac{\kappa^{2} T_{p} \alpha^{2}}{4 \sqrt{2}(p-3) ! \pi^{2}} \epsilon^{\alpha_{1} \cdots \alpha_{p-3} \beta_{1} \cdots \beta_{4}} c_{\alpha_{1} \cdots \alpha_{p-3}} k_{3 \beta_{1}} k_{4 \beta_{3}} \\
& {\left[\left(k_{4} \cdot S \cdot \zeta_{3 \beta_{2}}\right)\left(k_{3} \cdot \zeta_{4 \beta_{4}}\right)-\left(k_{3} \cdot S \cdot k_{4}\right)\left(\zeta_{3 \beta_{2}} \cdot \zeta_{4 \beta_{4}}\right)+\left(k_{4} \cdot \zeta_{3 \beta_{2}}\right)\left(k_{3} \cdot S \cdot \zeta_{4 \beta_{4}}\right)\right.} \\
& \left.-\left(k_{3} \cdot k_{4}\right)\left(\zeta_{3 \beta_{2}} \cdot S \cdot \zeta_{4 \beta_{4}}\right)\right] \\
& \int_{\left|z_{3}\right|,\left|z_{4}\right|>1} d^{2} z_{3} d^{2} z_{4}\left(\left|z_{3}\right|^{2}-1\right)^{k_{3} \cdot S \cdot k_{3}}\left(\left|z_{4}\right|^{2}-1\right)^{k_{4} \cdot S \cdot k_{4}}\left|z_{3}\right|^{-2 k_{3} \cdot S \cdot k_{3}-2 k_{3} \cdot S \cdot k_{4}-2} \\
& \left|z_{4}\right|^{-2 k_{4} \cdot S \cdot k_{4}-2 k_{3} \cdot S \cdot k_{4}-2}\left|z_{3}-z_{4}\right|^{2 k_{3} \cdot k_{4}-2}\left|z_{3} \bar{z}_{4}-1\right|^{2 k_{3} \cdot S \cdot k_{4}-2}\left(z_{3} \bar{z}_{4}-\bar{z}_{3} z_{4}\right)^{2} .
\end{aligned}
$$

This is the general result. However, when we want to talk about the worldvolume action of a D-brane, we might be especially interested in momenta and polarizations which are along the brane worldvolume. From now on we specialize to that case. Because of the mass shell condition and momentum conservation in the worldvolume directions the integral above simplifies to

$$
\mathcal{I}=\int_{\left|z_{3}\right|,\left|z_{4}\right|>1} d^{2} z_{3} d^{2} z_{4}\left|z_{3} z_{4}\right|^{-2}\left|z_{3}-z_{4}\right|^{-2}\left|z_{3} \bar{z}_{4}-1\right|^{-2}\left(z_{3} \bar{z}_{4}-\bar{z}_{3} z_{4}\right)^{2}
$$

The integral depends only on the difference of the phases of $z_{3}$ and $z_{4}$, so one of the angular integrations just gives a factor of $2 \pi$. By changing variables to the cosine of the difference of the phases, it can be verified that the second angular integration results in a hypergeometric function. With $r=\left|z_{3}\right|, t=\left|z_{4}\right|, x=\frac{4 r t}{(r+t)^{2}}$ and $y=\frac{4 r t}{(r t+1)^{2}}$ the integral becomes (up to a minus sign) [8]:

$$
\mathcal{I}=\pi^{2} \int_{r, t>1} d r d t(r t)^{-1} x y F_{1}\left(\frac{3}{2}, 1,1,3 ; x, y\right)
$$


Performing a change of integration variables and manipulating the hypergeometric function, this becomes

$$
\mathcal{I}=\frac{\pi^{2}}{2} \int_{0}^{1} d y(1-y)^{-1 / 2} \sum_{n=0}^{\infty} \frac{\left(\frac{3}{2}\right)_{n}}{(3)_{n}} y^{n} \int_{0}^{1} d x(1-x)^{-1 / 2} F\left(\frac{3}{2}+n, 1,3+n ; x\right)
$$

In this expression, both integrals are well-known [8]. The result is

$$
\mathcal{I}=\frac{\pi^{3}}{2} \sum_{k, n=0}^{\infty} \frac{\left(\frac{3}{2}\right)_{n+k} \Gamma(k+1) \Gamma(n+1)}{(3)_{n+k} \Gamma\left(k+\frac{3}{2}\right) \Gamma\left(n+\frac{3}{2}\right)}=\frac{4 \pi^{4}}{3} .
$$

The scattering amplitude thus becomes

$$
\mathcal{A}=\frac{\sqrt{2} \pi^{2} \kappa^{2} T_{p} \alpha^{\prime 2}}{3(p-3) !} \epsilon^{\alpha_{1} \cdots \alpha_{p-3} \beta_{1} \cdots \beta_{4}} c_{\alpha_{1} \cdots \alpha_{p-3}} k_{3 \beta_{1}} k_{4 \beta_{3}}\left(k_{4} \cdot \zeta_{3 \beta_{2}}\right)\left(k_{3} \cdot \zeta_{4 \beta_{4}}\right)
$$

\section{D-brane action}

Let us compare these results to what one expects from the D-brane action in supergravity.

As to the amplitude in Section 2, the relevant part of the D-brane action is (see Eq. (A.51))

$$
\frac{T_{p}}{\kappa} \int_{p+1} \hat{\mathcal{C}}_{p-1}^{\prime} \wedge \hat{B}^{\prime}
$$

The amplitude calculated from this interaction thus becomes

$$
\mathcal{A}_{\text {sugra }}=\frac{\kappa T_{p}}{(p-1) !} \epsilon^{\alpha_{1} \ldots \alpha_{p-1} \beta_{1} \beta_{2}} c_{\alpha_{1} \ldots \alpha_{p-1}} \zeta_{\beta_{1} \beta_{2}}
$$

This is twice the string result. However, as suggested by R. Russo, another diagram should be considered in the supergravity computation. In fact, the bulk supergravity action given in the appendix is not the whole story: the field strength of a $p+1$-form $\mathrm{RR}$ potential gets a correction involving the $p-1$-form $\mathrm{RR}$ potential and the NS 2-form. This implies that there is a diagram in which the D-brane emits an intermediate $p+1$-form potential. This contribution should be added to the contact amplitude given above. Checking the structure and the coefficient of this contribution shows that this could explain the apparent mismatch.

For the amplitude in Section 3 the relevant part of the action is

$$
\frac{T_{p}}{\kappa} \int_{p+1} \hat{\mathcal{C}}_{p-3}^{\prime} \wedge \frac{1}{2} \frac{\left(4 \pi \alpha^{\prime}\right)^{2}}{192 \pi^{2}} \operatorname{tr}(\hat{R} \wedge \hat{R})
$$

\footnotetext{
** We thank Walter Troost for showing us how to sum the following series.
} 
Expanding $\hat{R} \wedge \hat{R}$ and writing everything in terms of canonically normalized fields (see Eq. (A.53) and Eq. (A.56)) one obtains for the scattering amplitude in supergravity

$$
\mathcal{A}_{\text {sugra }}=\frac{\sqrt{2} \pi^{2} \kappa^{2} T_{p} \alpha^{2}}{6(p-3) !} \epsilon^{\alpha_{1} \cdots \alpha_{p-3} \beta_{1} \cdots \beta_{4}} c_{\alpha_{1} \cdots \alpha_{p-3}} k_{3 \beta_{1}} k_{4 \beta_{3}}\left(k_{4} \cdot \zeta_{3 \beta_{2}}\right)\left(k_{3} \cdot \zeta_{4 \beta_{4}}\right)
$$

This time, the supergravity computation seems to be missing a factor of 2 relative to the string result. Again, extra contributions might come from modifications of the RR field strengths [1]. However, the details of this possible solution of the mismatch are left for future research.

\section{Orientifold planes}

The computations in the previous sections were done in oriented string theory. From the (oriented) type IIB theory, one can construct the (unoriented) type I theory by modding out worldsheet parity. This operation makes another kind of objects enter the story: the orientifold planes [9]. In Ref. [3] it was argued that orientifold planes exhibit a coupling to gravity similar to the one we computed for D-branes in Section 3. Moreover, it was claimed that the orientifold 9-plane in type I theory couples with half the strength of the 32 type IIB D-branes (providing the Chan-Paton factors [10]) together.

As remarked in Ref. [3], the presence of that interaction can be checked by computing an $R P^{2}$ diagram with three insertions. This is analogous to the computation for D-branes: rather than cutting a hole in the sphere to obtain a disk, one inserts a crosscap. For the case of all Neumann boundary conditions, the 'crosscap state' representing the insertion of a crosscap was discussed in Ref. [11]: apart from a different normalization of $R P^{2}$ relative to disk amplitudes (giving an extra factor of 32 ), the crosscap operator is obtained by inserting $(-1)^{n}$ in every term of the exponent of the boundary state.

The computation of the $R P^{2}$ diagram is very similar to the one in Section 3 . Because of the $(-1)^{n}$ insertions, every $z_{i} \bar{z}_{j}$ combination gets replaced by $-z_{i} \bar{z}_{j}$. In Eq. (3.14) this amounts to replacing the 1 by -1 . Analogous calculations to the ones which led to Eq. (3.17) result in

$$
\mathcal{I}_{\text {crosscap }}=\frac{2 \pi^{4}}{3}
$$

half the result of the D-brane computation. Taking into account the factor of 32 from the crosscap normalization, this reproduces precisely the coupling predicted in Ref. [3]. 


\section{Discussion}

By evaluating the appropriate string diagrams we have derived two specific terms of the D-brane effective action. This nicely illustrates how superstring theory provides its own consistency. If (intersecting) D-branes are to be included into the picture then from Ref. [1] the terms in the effective D-brane action have to be there for the low energy theory on the brane not to suffer from anomalies.

Repeating the analysis for the orientifold, we have extended our results to the orientifold $p_{1}(R)$ coupling, first proposed in Ref. [3]. Thus new evidence has been

provided for the relative factor $\frac{1}{2}$ between the D-brane and orientifold couplings.

Applying the above machinery one could in principle go beyond our results. The fourth order curvature term can be treated analogously but may turn out to be a hard nut to crack, though.

From a more general perspective one may ask the following question: is there room for a similar gravitational coupling in the worldvolume theory of the M-theory 5-brane [12]? In Ref. [13] Sen found such a coupling in the worldvolume action of the KK-monopole in M-theory. It would be interesting to see whether his procedure can be applied to the M5-brane.

\section{Acknowledgments}

We would like to thank Marco Billó, Andrea Pasquinucci, Rodolfo Russo and Walter Troost for very helpful discussions. This work was supported by the European Commission TMR programme ERBFMRX-CT96-0045.

\section{Note added}

While this paper was circulating as a preprint, the issue of gravitational couplings on the M5-brane was addressed in Ref. [19]. 


\section{A Conventions and normalizations}

We normalize the boundary state as in [6]:

$$
|B, \eta\rangle_{\mathrm{R}, \mathrm{NS}}=\frac{T_{p}}{2}\left|B_{X}\right\rangle\left|B_{\mathrm{gh}}\right\rangle\left|B_{\psi}, \eta\right\rangle_{\mathrm{R}, \mathrm{NS}}\left|B_{\mathrm{sgh}}, \eta\right\rangle_{\mathrm{R}, \mathrm{NS}}
$$

where

$$
\begin{gathered}
\left|B_{X}\right\rangle=\delta^{\left(d_{\perp}\right)}(\hat{q}-y) \exp \left[-\sum_{n=1}^{\infty} \frac{1}{n} \alpha_{-n} \cdot S \cdot \tilde{\alpha}_{-n}\right]|0 ; k=0\rangle, \\
\left|B_{\mathrm{gh}}\right\rangle=\exp \left[\sum_{n=1}^{\infty}\left(c_{-n} \tilde{b}_{-n}-b_{-n} \tilde{c}_{-n}\right)\right] \frac{c_{0}+\tilde{c}_{0}}{2}|q=1\rangle|\tilde{q}=1\rangle,
\end{gathered}
$$

and, in the NS sector in the $(-1,-1)$ picture,

$$
\begin{gathered}
\left|B_{\psi}, \eta\right\rangle_{\mathrm{NS}}=\exp \left[\mathrm{i} \eta \sum_{m=1 / 2}^{\infty} \psi_{-m} \cdot S \cdot \tilde{\psi}_{-m}\right]|0\rangle \\
\left|B_{\mathrm{sgh}}, \eta\right\rangle_{\mathrm{NS}}=\exp \left[\mathrm{i} \eta \sum_{m=1 / 2}^{\infty}\left(\gamma_{-m} \tilde{\beta}_{-m}-\beta_{-m} \tilde{\gamma}_{-m}\right)\right]|P=-1\rangle|\tilde{P}=-1\rangle,
\end{gathered}
$$

or, in the $\mathrm{R}$ sector in the $(-1 / 2,-3 / 2)$ picture,

$$
\begin{array}{r}
\left|B_{\psi}, \eta\right\rangle_{\mathrm{R}}=\exp \left[\mathrm{i} \eta \sum_{m=1}^{\infty} \psi_{-m} \cdot S \cdot \tilde{\psi}_{-m}\right]\left|B_{\psi}, \eta\right\rangle_{\mathrm{R}}^{(0)} \\
\left|B_{\mathrm{sgh}}, \eta\right\rangle_{\mathrm{R}}=\exp \left[\mathrm{i} \eta \sum_{m=1}^{\infty}\left(\gamma_{-m} \tilde{\beta}_{-m}-\beta_{-m} \tilde{\gamma}_{-m}\right)\right]\left|B_{\mathrm{sgh}}, \eta\right\rangle_{\mathrm{R}}^{(0)},
\end{array}
$$

where, if we define

$$
\mathcal{M}^{(\eta)}=C \Gamma^{0} \Gamma^{l_{1}} \ldots \Gamma^{l_{p}}\left(\frac{1+\mathrm{i} \eta \Gamma_{11}}{1+\mathrm{i} \eta}\right)
$$

the zero mode parts of the boundary state are

$$
\begin{gathered}
\left|B_{\psi}, \eta\right\rangle_{\mathrm{R}}^{(0)}=\mathcal{M}_{A B}^{(\eta)}|A\rangle|\tilde{B}\rangle \\
\left|B_{\mathrm{sgh}}, \eta\right\rangle_{\mathrm{R}}^{(0)}=\exp \left[\mathrm{i} \eta \gamma_{0} \tilde{\beta}_{0}\right]|P=-1 / 2\rangle|\tilde{P}=-3 / 2\rangle .
\end{gathered}
$$

The matrix $S_{\mu \nu}$ is given by

$$
S_{\mu \nu}=\left(\eta_{\alpha \beta},-\delta_{i j}\right)
$$

The overall normalization factor $T_{p}$ is claimed to be fixed from factorization of amplitudes of closed strings emitted from a disk [14, 7]. It is the tension of the $\mathrm{D} p$-brane

$$
T_{p}=\sqrt{\pi}\left(2 \pi \sqrt{\alpha^{\prime}}\right)^{3-p}
$$


The GSO projection acts as follows:

$$
\begin{aligned}
|B\rangle_{\mathrm{NS}} & =\frac{1}{2}\left(|B,+\rangle_{\mathrm{NS}}-|B,-\rangle_{\mathrm{NS}}\right), \\
|B\rangle_{\mathrm{R}} & =\frac{1}{2}\left(|B,+\rangle_{\mathrm{R}}+|B,-\rangle_{\mathrm{R}}\right) .
\end{aligned}
$$

All this is explained in more detail in [6]. Conventions on the RR zero modes and gamma matrices can be found in the Appendix of [7]. Here we only note that

$$
\left(\Gamma^{\mu}\right)^{T}=-C \Gamma^{\mu} C^{-1}
$$

and

$$
\begin{aligned}
\psi_{0}^{\mu}|A\rangle|\widetilde{B}\rangle & =\frac{1}{\sqrt{2}}\left(\Gamma^{\mu}\right)_{C}^{A}(11)_{D}^{B}|C\rangle|\widetilde{D}\rangle \\
\widetilde{\psi}_{0}^{\mu}|A\rangle|\widetilde{B}\rangle & =\frac{1}{\sqrt{2}}\left(\Gamma_{11}\right)_{C}^{A}\left(\Gamma^{\mu}\right)_{D}^{B}|C\rangle|\widetilde{D}\rangle
\end{aligned}
$$

In our computation we have put

$$
\alpha^{\prime}=2
$$

so that the following OPEs hold [15]:

$$
\begin{aligned}
X^{\mu}(z) X^{\nu}(w) & =-\eta^{\mu \nu} \log (z-w) \\
\psi^{\mu}(z) \psi^{\nu}(w) & =-\eta^{\mu \nu}(z-w)^{-1}
\end{aligned}
$$

where

$$
X(z, \bar{z})=X(z)+X(\bar{z}) .
$$

Expanding the fields in modes, this gives

$$
\begin{aligned}
{\left[\alpha_{m}^{\mu}, \alpha_{n}^{\nu}\right] } & =\eta^{\mu \nu} m \delta_{m+n} \\
{\left[\alpha_{0}^{\mu}, q^{\nu}\right] } & =-\mathrm{i} \eta^{\mu \nu} \\
\left\{\psi_{m}^{\mu}, \psi_{n}^{\nu}\right\} & =\eta^{\mu \nu} \delta_{m+n},
\end{aligned}
$$

where

$$
\begin{aligned}
\mathrm{i} \partial X^{\mu}(z) & =\sum_{n} z^{-n-1} \alpha_{n}^{\mu}, \\
\mathrm{i} \psi^{\mu}(z) & =\sum_{r} z^{-r-\frac{1}{2}} \psi_{r}^{\mu} .
\end{aligned}
$$

For the graviton vertex operator in the $(-1,-1)$ picture we take

$$
V_{g}^{(-1)}=\zeta_{\mu \nu} \psi^{\mu} \tilde{\psi}^{\nu} e^{\mathrm{i} k \cdot X}
$$


This normalization is such that

$$
\left\langle V_{g}^{(-1)} \mid V_{g}^{(-1)}\right\rangle=-\operatorname{tr} \zeta^{2}
$$

It can be checked [7] that, with this normalization,

$$
\left\langle 0\left|V_{g}^{(-1)}\right| B\right\rangle_{\mathrm{NS}}
$$

reproduces the one point function of the canonically normalized graviton in supergravity, where the D-brane is described by the DBI action. The canonically normalized graviton field is obtained by expanding

$$
g_{\mu \nu}=\eta_{\mu \nu}+2 \kappa h_{\mu \nu}
$$

(where $\kappa=8 \pi^{7 / 2} \alpha^{\prime 2} g_{s}=\sqrt{8 \pi G_{N}}$ ) in the bulk supergravity action in Einstein frame

$$
\begin{aligned}
& S_{\mathrm{IIA}, \mathrm{B}}=- \frac{1}{2 \kappa^{2}} \int d^{10} x \sqrt{-g}\left[R+\frac{1}{2}(d \Phi)^{2}+\frac{1}{12} e^{-\Phi}\left(d B^{\prime}\right)^{2}\right. \\
&\left.+\sum \frac{1}{2(p+2) !} e^{(3-p) \Phi / 2}\left(d \mathcal{C}_{p+1}^{\prime}\right)^{2}\right]
\end{aligned}
$$

(see, for instance [16]). The DBI action is given in string frame $\left(G_{\mu \nu}=e^{\Phi / 2} g_{\mu \nu}\right)$ by

$$
S_{B I}=-\frac{T_{p}}{\kappa} \int d^{p+1} \xi e^{-\Phi} \sqrt{-\operatorname{det}\left[\hat{G}_{\alpha \beta}+\hat{B}_{\alpha \beta}^{\prime}+2 \pi \alpha^{\prime} F_{\alpha \beta}\right]}
$$

(Here $\hat{G}$ denotes the pullback to the D brane worldvolume of the bulk field $G$ etc.) In addition, the D-brane action contains a WZ part

$$
S_{W Z}=\frac{T_{p}}{\kappa} \int_{p+1} \hat{\mathcal{C}}^{\prime} \wedge e^{2 \pi \alpha^{\prime} F+\hat{B}^{\prime}} \wedge \sqrt{\hat{A}(\hat{R})}
$$

where

$$
\hat{A}(\hat{R})=1+\frac{\left(4 \pi \alpha^{\prime}\right)^{2}}{192 \pi^{2}} \operatorname{tr}(\hat{R} \wedge \hat{R})+\ldots
$$

These actions can be written in terms of canonically normalized bulk fields by substituting

$$
\begin{aligned}
G & =e^{\Phi / 2}\left(\eta_{\mu \nu}+2 \kappa h_{\mu \nu}\right) \\
\Phi & =\sqrt{2} \kappa \chi \\
B^{\prime} & =\sqrt{2} \kappa e^{\Phi / 2} B \\
\mathcal{C}^{\prime} & =\sqrt{2} \kappa \mathcal{C} .
\end{aligned}
$$

For the normalization of the graviton vertex operator in the $(0,0)$ picture, we follow Section 3.4 of [17]:

$$
V_{g}^{(0)}=\frac{\kappa}{\pi} \zeta_{\mu \nu}\left(\partial X^{\mu}+\mathrm{i} k \cdot \psi \psi^{\mu}\right)\left(\bar{\partial} X^{\nu}+\mathrm{i} k \cdot \tilde{\psi} \tilde{\psi}^{\nu}\right) e^{\mathrm{i} k \cdot X}
$$


(where we put $\alpha^{\prime}=2$ rather than $\alpha^{\prime}=1 / 2$ as in [17]).

For the Kalb-Ramond field we would correspondingly find

$$
V_{B}^{(0)}=\frac{\kappa}{\sqrt{2} \pi} \zeta_{\mu \nu}\left(\partial X^{\mu}+\mathrm{i} k \cdot \psi \psi^{\mu}\right)\left(\bar{\partial} X^{\nu}+\mathrm{i} k \cdot \tilde{\psi} \tilde{\psi}^{\nu}\right) e^{\mathrm{i} k \cdot X}
$$

(The factor of $1 / \sqrt{2}$ is due to the different rescaling between the canonically normalized fields and the fields appearing in the string action. The $\zeta_{\mu \nu}$ in the vertex operators correspond to polarizations of canonically normalized fields.)

Finally, we introduce a (canonically normalized) out-state for a RR-potential 61:

$$
\left\langle\mathcal{C}_{m+1}\right|=\frac{1}{2}\left(\left\langle\mathcal{C}_{m+1},+\right|+\left\langle\mathcal{C}_{m+1},-\right|\right)
$$

with

$$
\left\langle\mathcal{C}_{m+1}, \eta\right|=e^{i \eta \beta_{0} \tilde{\gamma}_{0}} \mathcal{N}_{A B-3 / 2}\langle A|-1 / 2\langle\tilde{B}|
$$

where we defined

$$
\mathcal{N}_{A B}=\frac{\left(C \Gamma^{\mu_{1} \cdots \mu_{m+1}}\right)_{A B}}{2 \sqrt{2}(m+1) !} c_{\mu_{1} \cdots \mu_{m+1}} .
$$

(Here $c$ denotes the polarization tensor of $\mathcal{C}$ ). The factor $\frac{1}{2 \sqrt{2}}$ is justified by computing

$$
\left\langle\mathcal{C}_{m+1} \mid \mathcal{C}_{m+1}\right\rangle=\frac{1}{(m+1) !} c_{\mu_{1} \cdots \mu_{m+1}} c^{\mu_{1} \cdots \mu_{m+1}}
$$

As a check, one can derive [18] the $e^{2 \pi \alpha^{\prime} F}$ part of the WZ term of the D-brane action by projecting the states $\left\langle\mathcal{C}_{p+1-2 n}\right|, n=0,1, \ldots$ on the boundary state with $F$ turned on.

So far we have only computed one-point functions in the presence of a D-brane. The ghost zero modes have been taken into account by not integrating over the position of the inserted vertex operator. Because of the peculiar ghost structure of the boundary state, it is not so clear how the ghosts should be treated when one inserts more than one vertex operator (i.e. which part of the $S L(2)$ group volume still has to be divided out). The procedure we will follow (and motivate below) consists of integrating over the positions of all vertex operators except the one inserted at infinity.

To motivate this procedure, we compute the two graviton amplitude for both polarizations along the brane (up to powers of i):

$$
\left\langle 0\left|V_{g}^{(-1)} V_{g}^{(0)}\right| B\right\rangle_{\mathrm{NS}}=\kappa T_{p} q^{2} \operatorname{tr}\left(\zeta_{1} \cdot \zeta_{2}\right) B\left(-\frac{t}{2}, 2 q^{2}+1\right)
$$

where $q^{2}=\frac{1}{2}\left(k_{2} \cdot S \cdot k_{2}\right)$ and $t=-\left(k_{1}+k_{2}\right)^{2}$. This is the correct result (see for instance [5]). 


\section{References}

[1] M. Green, J. Harvey and G. Moore, I-brane inflow and anomalous couplings on D-branes, Class. Quant. Grav. 14 (1997) 47, hep-th/9605033.

[2] M. Bershadsky, V. Sadov and C. Vafa, D-branes and topological field theories, Nucl. Phys. B463 (1996) 420, hep-th/9511222.

[3] K. Dasgupta, D. Jatkar and S. Mukhi, Gravitational couplings and $Z_{2}$ orientifolds, Nucl. Phys. B523 (1998) 465, hep-th/9707224.

K. Dasgupta and S. Mukhi, Anomaly inflow on orientifold planes, J. High Energy Phys. 3 (1998) 4, hep-th/9709219

[4] I. Klebanov and L. Thorlacius, The Size of p-Branes, Phys. Lett. B371 (1996) 51-56, hep-th/9510200.

[5] M. Garousi and R. Myers, Superstring Scattering from D-Branes, Nucl. Phys. B475 (1996) 193, hep-th/9603194.

[6] M. Billó, P. Di Vecchia, M. Frau, A. Lerda, I. Pesando, R. Russo and S. Sciuto, Microscopic string analysis of the D0-D8 brane system and dual $R-R$ states, hep-th/9802088.

[7] P. Di Vecchia, M. Frau, I. Pesando, S. Sciuto, A. Lerda, R. Russo, Classical p-branes from boundary state, Nucl. Phys. B507 (1997) 259, hep-th/9707068.

[8] I. Gradshteyn and I. Ryzhik, Table of Integrals, Series, and Products, Academic Press 1965.

[9] A. Sagnotti, Open strings and their symmetry groups, in Non-perturbative Quantum Field Theory, Cargese 1987, eds G.Mack et al, Pergamon Press 1988.

P. Horava, Background duality of open string models, Phys. Lett. B231 (1989) 251.

J. Dai, R.G. Leigh and J. Polchinski, New connections between string theories, Mod. Phys. Lett. A4 (1989) 2073.

[10] J. Polchinski and E. Witten, Evidence for heterotic-type I string duality, Nucl. Phys. B460 (1996) 525, hep-th/9510169.

[11] C. Callan, C. Lovelace, C. Nappi and S. Yost, Adding holes and crosscaps to the superstring, Nucl. Phys. B293 (1987) 83.

[12] P. Pasti, D. Sorokin and M. Tonin, Covariant action for a D=11 five-brane with the chiral field, Phys. Lett. 398B (1997) 41, hep-th/9701037.

M. Aganagic, J. Park, C. Popescu and J. Schwarz, World volume action of the $M$ theory five-brane, Nucl. Phys. B496 (1997) 191, hep-th/9701166. 
[13] A. Sen, Strong Coupling Dynamics of Branes from M-theory, J.High Energy Phys. 10 (1997) 002, hep-th/9708002.

[14] M. Frau, I. Pesando, S. Sciuto, A. Lerda and R. Russo, Scattering of Closed Strings from Many D-branes, Phys. Lett. 400B (1997) 52, hep-th/9702037.

[15] D. Friedan, Notes on String Theory and Two Dimensional Conformal Field Theory, in Unified String Theories, eds M. Green and D. Gross, World Scientific 1986.

[16] C. Bachas, Lectures on D-branes, hep-th/9806199.

[17] M. Green, J. Schwarz and E. Witten, Superstring Theory, Vol. 1, Cambridge University Press 1987.

[18] P. Di Vecchia, unpublished notes.

[19] S. Mukhi, Dualities and the $S L(2, Z)$ Anomaly, hep-th/9810213. 